\title{
Risk and Healthcare Costs of Chemotherapy- Induced Neutropenic Complications in Women with Metastatic Breast Cancer
}

\author{
Derek Weycker $^{\mathrm{a}}$ John Edelsberg ${ }^{\mathrm{a}} \quad$ Alex Kartashov $^{\mathrm{a}}$ Rich Barron $^{\mathrm{b}}$ Gary Lyman ${ }^{\mathrm{c}}$ \\ a Policy Analysis Inc. (PAI), Brookline, Mass., ${ }^{\mathrm{b}}$ Amgen Inc., Thousand Oaks, Calif., and ${ }^{\mathrm{C} C e n t e r}$ for Clinical Health \\ Policy Research, Duke University School of Medicine, Durham, N.C., USA
}

\section{Key Words}

Metastatic breast cancer - Febrile neutropenia $\cdot$ Costs and cost analysis

\begin{abstract}
Background: The burden of chemotherapy-induced neutropenic complications (CINC) in women with metastatic breast cancer (MBC) is largely unknown and may differ across cancer populations due to variation in the characteristics of patients, their disease and their treatment. Methods: This study employed a retrospective cohort design and US healthcare claims data (2003-2009). For each woman in the study database who received myelotoxic chemotherapy for $M B C$, the first observed course and each cycle within the course were characterized. Risk and healthcare costs of CINC - by care setting - were descriptively analyzed on an overall basis by chemotherapy cycle and chemotherapy regimen. Results: Among 2,620 study subjects, most received chemotherapy with cyclophosphamide/doxorubicin (25\%), docetaxel $(20 \%)$ or paclitaxel (12\%). Thirty-one percent of subjects received colony-stimulating factors (CSF) prophylactically in their first chemotherapy cycle and an additional $13 \%$ first received CSF prophylaxis after cycle one. CINC developed in $11 \%$ of subjects; among these subjects, $88 \%$ required inpatient care and $45 \%$ experienced CINC in the first
\end{abstract}

cycle of chemotherapy. For CINC requiring inpatient care, costs averaged USD 12,869 (95\% Cl: USD 12,622-13,116), and for CINC requiring outpatient care only, USD 2,030 (CI: USD 1,925-2,135). Conclusion: CINC is a clinically and economically important threat among women with $\mathrm{MBC}$, and should be an important consideration in the treatment of this population.

Copyright $\odot 2012$ S. Karger AG, Basel

\section{Introduction}

Breast cancer is the most common form of cancer among women in the US, and the second leading cause of cancer death [1]. It is estimated that 1 in every 8 women will develop breast cancer during her lifetime [1]. In 2009, an estimated 192,370 women were diagnosed with breast cancer, and 40,170 women died from the disease [1]. Approximately $6 \%$ of women with breast cancer have metastatic disease at initial presentation and an additional 20 $40 \%$ of breast cancer patients develop metastatic disease at some point following diagnosis, often many years later. Median survival in women with metastatic breast cancer (MBC) is about $18-24$ months, but many patients survive several years [2]. 
$\mathrm{MBC}$ is a heterogeneous disease that is managed with a variety of treatment modalities. Approximately two thirds of women with $\mathrm{MBC}$ have tumors that are hormone-receptor-positive (i.e. they express estrogen and/or progesterone receptors) [3]; endocrine therapy is appropriate for the great majority of these patients. Between 20 and 30\% of patients with breast cancer have tumors that express HER2/neu (HER-2), a tyrosine kinase growth factor receptor located on cell membranes $[4,5]$. Targeted therapy with the monoclonal antibody, trastuzumab, or the dual tyrosine kinase inhibitor, lapatinib, in combination with hormonal and/or conventional chemotherapy, has been reported to improve response rates in these patients $[5,6]$. For patients with tumors that do not express hormone receptors and those with HER-2-negative tumors, chemotherapy remains the main treatment option [7]. Chemotherapy also is the primary treatment modality for patients with rapidly progressive visceral disease and those with hormonereceptor-positive tumors that do not respond or have become resistant to endocrine therapy $[8,9]$.

Neutropenia is a common side effect of myelosuppressive chemotherapy. Neutropenia increases the risk of infection, which is typically signaled by fever. When neutropenic patients develop fever, i.e. febrile neutropenia (FN), the likelihood of infection and serious consequences often necessitates hospitalization for urgent evaluation, ongoing monitoring and administration of intravenous antibiotics $[10,11]$. FN, as well as severe or prolonged neutropenia, can lead to dose delays, dose reductions, and/or chemotherapy discontinuations, interfering with the delivery of optimal treatment and possibly adversely affecting patient outcomes [11-14].

In several recent randomized trials of the efficacy and safety of combination versus sequential-agent chemotherapy in women with $\mathrm{MBC}$, the risk of $\mathrm{FN}$ has been reported to be as high as $48 \%$, although for many chemotherapy regimens, the risk of $\mathrm{FN}$ has been reported to be much lower [15-18]. Because the intent of chemotherapy is typically palliative in this patient population, the incidence of toxicities such as FN is an important consideration in the selection of chemotherapy regimens and, along with overall survival, should be considered as one of the key endpoints in making treatment decisions [15]. Moreover, because the benefits of chemotherapy for $\mathrm{MBC}$ - in terms of both extensions in life expectancy and an enhanced quality of life - are typically limited, the cost of FN and other associated toxicities is an especially important consideration in an era of increased emphasis on achieving an acceptable balance between the costs and benefits of medical interventions [16-20].
While several studies have examined the risk of chemotherapy-induced neutropenic complications (CINC) and the associated economic consequences among patients with various types of cancer in clinical practice $[10$, 21-23], in proportion to the prevalence of MBC, little is known about these measures for women with this condition in real-world settings. Besides having more advanced disease - on average - versus a general population of cancer patients, women with MBC are likely to receive different chemotherapy regimens and chemotherapy dose intensity, are more likely to have a history of chemotherapy and/or radiation therapy, bone marrow involvement, chronic comorbidities, poor performance status, and to be older, all of which may impact the risk and economic burden of CINC.

\section{Patients and Methods}

\section{Data Source}

Data spanning January 1, 2003 to December 31, 2009 were obtained from 2 large U.S. healthcare claims databases, and patientlevel claims information was pooled for analyses. Analytic files comprising data for all patients and all variables required for analyses were created from each database, and variable names were standardized between files for purposes of integration. Only observed data were utilized in defining study variables and study measures.

The 2 databases - the Thomson Reuters MarketScan Commercial Claims and Encounters and Medicare Supplemental and Coordination of Benefits Database (MarketScan Database) and the Intercontinental Marketing Services LifeLink Database (LifeLink Database) - both comprise medical (i.e. facility and professional service) and outpatient pharmacy claims from a large number of participating private health plans. The MarketScan Database includes healthcare claims information from more than 50 employer-sponsored health insurance plans providing coverage via fee-for-service and fully/partially capitated products, including health maintenance organizations ( $16 \%$ of all patients in the study database, i.e. cancer chemotherapy patients), preferred/exclusive provider organizations (52\%), point of service (10\%) and indemnity (17\%). Healthcare claims information in the MarketScan Database is available for approximately 15 million people annually, including active employees, early retirees, Consolidated Omnibus Budget Reconciliation Act continuees, and their dependants, as well as retirees with employer-sponsored Medicare supplemental coverage. Approximately $43 \%$ of people in the study database are aged $18-49$ years, $35 \%$ are $50-64$ years, and $22 \%$ are $\geq 65$ years; $42 \%$ reside in the South region of the US, followed by the Midwest (26\%), West (21\%), and Northeast (11\%). The LifeLink Database includes healthcare claims information from over 75 private US health plans providing healthcare coverage through fee-for-service and fully/partially capitated products, including health maintenance organizations ( $25 \%$ of patients in the study database), preferred/exclusive provider organizations (45\%), point of service (16\%), and indemnity (4\%). Healthcare claims in- 
formation in the LifeLink Database is available for approximately 15 million people annually. Approximately $58 \%$ of people in the study database are aged $18-49$ years, $36 \%$ are aged $50-64$ years and $6 \%$ are aged $\geq 65$ years; $35 \%$ reside in the Midwest region of the US, followed by the Northeast (26\%), South (24\%) and West (15\%).

Data available for each facility and professional service claim include date and place of service, diagnoses, procedures performed/services rendered and quantity of services (professional service claims). Data available for each retail pharmacy claim include the drug dispensed, quantity dispensed, dispensing date and number of days supplied. Selected demographic and eligibility information is also available for persons in both databases. All data can be arrayed to provide a detailed chronology of all medical and pharmacy services used by each plan member over time.

Patient-identifying information was encrypted or removed from the study databases prior to their release to the study investigators, as set forth in the corresponding Data Use Agreements. The study databases were evaluated and certified by an independent third party to be in compliance with the Health Insurance Portability and Accountability Act of 1996 (HIPAA) statistical deidentification standards and to satisfy the conditions set forth in sections 164.514 a-blii of the HIPAA Privacy Rule regarding the determination and documentation of statistically deidentified data. Use of the study databases for health services research is therefore fully compliant with the HIPAA Privacy Rule and federal guidance on Public Welfare and the Protection of Human Subjects [24].

\section{Study Population}

Source Population

The source population comprised all women who, between January 1, 2004 and December 31, 2008, were aged $\geq 18$ years and initiated myelotoxic chemotherapy for MBC. The presence of MBC was ascertained on the basis of: (1) $\geq 2$ medical encounters with a diagnosis of breast cancer (ICD-9-CM 174.x) at least 30 days apart (a requirement of a diagnosis in 2 claims separated by at least 1 month was expected to be supportive of a confirmed diagnosis of breast cancer), (2) $\geq 2$ medical encounters with a diagnosis of the same distant secondary malignant neoplasm [ICD-9CM 196.2, 196.5, 196.6, 196.8, 197.xx-198.xx (excluding 198.81 and 198.2)] any time after the first medical encounter with a diagnosis of breast cancer and (3) a diagnosis of breast cancer noted in at least 1 claim for chemotherapy (consistent with 1 of the criteria used in Stokes et al. [25]).

Receipt of chemotherapy was ascertained any time after the first encounter with a diagnosis of distant secondary malignant neoplasm, or no earlier than 45 days prior to this date (to allow for the possible occurrence of chemotherapy codes immediately prior to those for metastatic disease), based on the presence of medical claims with a Healthcare Common Procedure Coding System (HCPCS) Level II code for a chemotherapy drug or an HCPCS Level I (i.e. CPT), ICD-9-CM or UB-92 code for the administration of chemotherapy. The date of initial receipt of chemotherapy was designated as the 'index date'.

Myelotoxic chemotherapy was limited to regimens including taxane agents (paclitaxel and docetaxel) and anthracycline agents (doxorubicin, epirubicin, daunorubicin, idarubicin, valrubicin and mitoxantrone), since the risk of complications is typically intermediate or higher for such regimens and chemotherapy regi- mens for over $80 \%$ of women with MBC include a taxane or anthracycline agent [26]. Receipt of these agents was ascertained during the 7-day period beginning on the index date.

Chemotherapy Courses, Cycles and Regimens

For each patient in the source population, each unique cycle within the course of chemotherapy was identified. The first cycle was defined as beginning with the date of initiation of chemotherapy (i.e. the index date) and ending with the first service date for the next administration of chemotherapy (as evidenced by a medical claim with a corresponding HCPCS, ICD-9-CM or UB92 code), occurring at least 14 days - but no more than 59 days after the index date. If a second chemotherapy cycle did not commence prior to day 60 or if there was evidence of the receipt of radiation therapy (based on medical claims with relevant HCPCS, ICD-9-CM or UB-92 codes) during this period, both the first cycle and course of chemotherapy were considered to have been completed 30 days after the beginning of the cycle or on the day prior to initiation of radiation therapy, whichever occurred first. The second and all subsequent cycles of chemotherapy were similarly defined.

Chemotherapy agents were ascertained on a cycle-specific basis based on a review of all HCPCS Level II codes on medical claims with service dates within 6 days of the start of each cycle of chemotherapy. Regimens were categorized based on the combination of agents received in the first cycle. For patients in the source population with multiple courses of chemotherapy during the period of interest, only the first one was considered in analyses.

Exclusionary Criteria

Patients were excluded from the source population if they had: (1) $\geq 2$ medical encounters at least 30 days apart with a diagnosis of another malignant neoplasm (ICD-9-CM 140.xx-208.xx), excluding malignant neoplasm of breast (174.x), local or distant secondary malignant neoplasm (196.x-199.x), malignant neoplasm of skin other than melanoma (173.xx) or another malignant neoplasm if it was the same site as the metastases (e.g. malignant neoplasm of liver [155.0] if there was evidence of metastasis to liver [197.7], as defined above), (2) any gaps in their eligibility for comprehensive medical and drug benefits during the 1-year ('pretreatment') period prior to the initiation of chemotherapy, (3) evidence of hematopoietic stem-cell transplantation prior to or during receipt of chemotherapy or (4) any pharmacy claims for myelotoxic chemotherapy drugs or colony-stimulating factors (CSF) agents (precise dates of administration could not be ascertained for drugs dispensed through retail pharmacies) during the 30-day period before or during the receipt of chemotherapy.

\section{Neutropenic Complications}

Episodes

Neutropenic complications comprised encounters for the treatment of neutropenia (ICD-9-CM 288.0) as well as encounters for the treatment of related conditions. The latter comprised infections (list available from the authors upon request) and fever (ICD-9-CM 780.6).

Identifying hospitalization for CINC was based on admission for inpatient care with a diagnosis (principal or secondary) of neutropenia, fever or infection. Hospitalizations were identified on a cycle-specific basis using facility inpatient claims with admission dates any time between day 6 and the last day of the che- 
motherapy cycle. Hospitalizations for any reason (i.e. all-cause) were also identified.

Ascertaining outpatient care for CINC was based on an ambulatory encounter with a diagnosis of neutropenia, fever or infection and an HCPCS Level I (i.e. CPT) code for IV administration of antimicrobial therapy. Such encounters that preceded a hospitalization during the same cycle of chemotherapy were not considered as outpatient episodes. Outpatient care episodes were identified between day 6 and the last day of the chemotherapy cycle.

\section{Burden}

Characterizing the clinical and economic burdens of CINC was based on the care setting. For such complications requiring inpatient care, the burden was characterized in terms of total days in hospital, total inpatient costs, total outpatient costs incurred after the hospitalization but within same cycle and total overall costs (i.e. total inpatient plus total outpatient costs).

For complications treated on an outpatient basis, the burden was characterized in terms of total outpatient costs from the date of the event to the end of the cycle. Outpatient costs comprised encounters with a diagnosis of neutropenia, fever or infection, as well as use/prescriptions for CSF agents and antimicrobial therapy. Direct healthcare costs were estimated by tallying total allowed or paid amounts on medical and pharmacy claims for encounters with a diagnosis of neutropenia, fever or infection as well as claims for selected drugs (i.e. CSF agents and antibiotic therapy) from the date of the event to the end of the cycle.

\section{Patient and Treatment Characteristics}

Characteristics of women with MBC were evaluated including: age, the presence of selected comorbidities (diabetes, cardiovascular, liver and renal disease), history of blood disorders (anemia, neutropenia or other), infection, hospitalization, radiation treatment and other treatment modalities, the site of metastases, the use of CSF, the chemotherapy regimen and the year in which it took place (post-MBC diagnosis).

Age was assessed as of the first day of the first cycle of chemotherapy. Chronic comorbidities and a history of blood disorders, infections, hospitalization (all-cause) and radiation treatment were assessed from the beginning of the 1-year pretreatment period to the first day of the first cycle of chemotherapy. Chronic comorbidities were identified on the basis of $\geq 1$ diagnosis codes on inpatient claims, $\geq 2$ diagnosis codes on outpatient claims (excluding those for laboratory services) on different days, $\geq 1$ procedure codes, and $\geq 1$ drug codes, as appropriate (online supplement). Blood disorders and infections were identified on the basis of $\geq 1$ diagnosis codes (on inpatient and/or outpatient claims) and $\geq 1$ drug codes, as appropriate.

Other treatment modalities included the receipt of endocrine therapy [presumably among women with tumors that are hormone-receptor positive (i.e. that express estrogen and/or progesterone receptors)] and trastuzumab/lapatinib (presumably among women with tumors that express HER-2). Receipt of such therapies was ascertained from the beginning of the 1-year pretreatment period to the first day of the first cycle of chemotherapy using corresponding drug codes.

Use of CSF (i.e. filgrastim, pegfilgrastim or sargramostim) was characterized in terms of the reason, timing and duration of administration (daily agents only), on a cycle-specific basis, among all subjects in the study population. Characterizing the reason for the use of these agents was based on the timing of their administration within the course and cycles of chemotherapy, as follows: primary prophylaxis - receipt on or before day 5 of cycle one, secondary prophylaxis - first receipt (as prophylaxis) on or before day 5 of cycle two or a subsequent cycle and treatment first receipt (in a given cycle) any time after cycle day 5 or receipt following the end of prophylaxis (defined as a gap of $>3$ days). The timing of the use of these agents - as prophylaxis and treatment - was depicted based on the day of first receipt during the course and cycle of administration. The duration of CSF use - as prophylaxis and treatment - was characterized based on temporal patterns of administration. Receipt of CSF was identified based on medical claims with relevant codes: filgrastim (J1440, J1441), pegfilgrastim (C9119, S0135, J2505) or sargramostim (J2820).

\section{Statistical Analyses}

Characteristics of MBC patients and their cancer and treatment were described on an overall basis and stratified by chemotherapy regimen. Characteristics of the chemotherapy regimens were described, including the number and duration of cycles. The use of CSF was described on an overall basis and among selected common chemotherapy regimens, in terms of the frequency of administration as primary prophylaxis, secondary prophylaxis and treatment, respectively, as well as the patterns and levels of use within the course and cycles among those receiving CSF. Categorical variables were reported as numbers and percentages; for continuous variables, means (standard deviations), ranges, medians and percentiles (25th and 75th) were reported.

The crude incidence of CINC requiring inpatient care and outpatient care only was descriptively analyzed over the entire chemotherapy course as well as on a cycle-specific basis. Risks were analyzed for all patients in the study population and patients receiving selected chemotherapy regimens. The burden of CINC was descriptively analyzed among patients developing this condition, and was summarized in terms of levels of the associated use of resources (e.g. hospitalizations and outpatient encounters) and expenditures during the cycle, considering all episodes collectively and by care setting. Risks and burden were estimated in a noncomparative fashion, and were summarized using means and percentages along with corresponding 95\% CIs (estimated using normal and binomial distributions, as appropriate).

\section{Results}

\section{Patient Characteristics}

A total of 130,726 adult women in the study database met our criteria for a diagnosis of breast cancer ( $\geq 30$ days apart). Among these women, 25,247 (19\%) met our criteria for a diagnosis of $\mathrm{MBC}$ and 4,695 (4\%) also initiated a course of myelotoxic chemotherapy (with a minimum first-cycle length of 14 days). The final study population included 2,620 women with $\mathrm{MBC}$ who met all remaining selection criteria (fig. 1).

Mean $( \pm$ SD) age of study subjects was $55( \pm 10)$ years, and the prevalence of each of the chronic comorbidities 


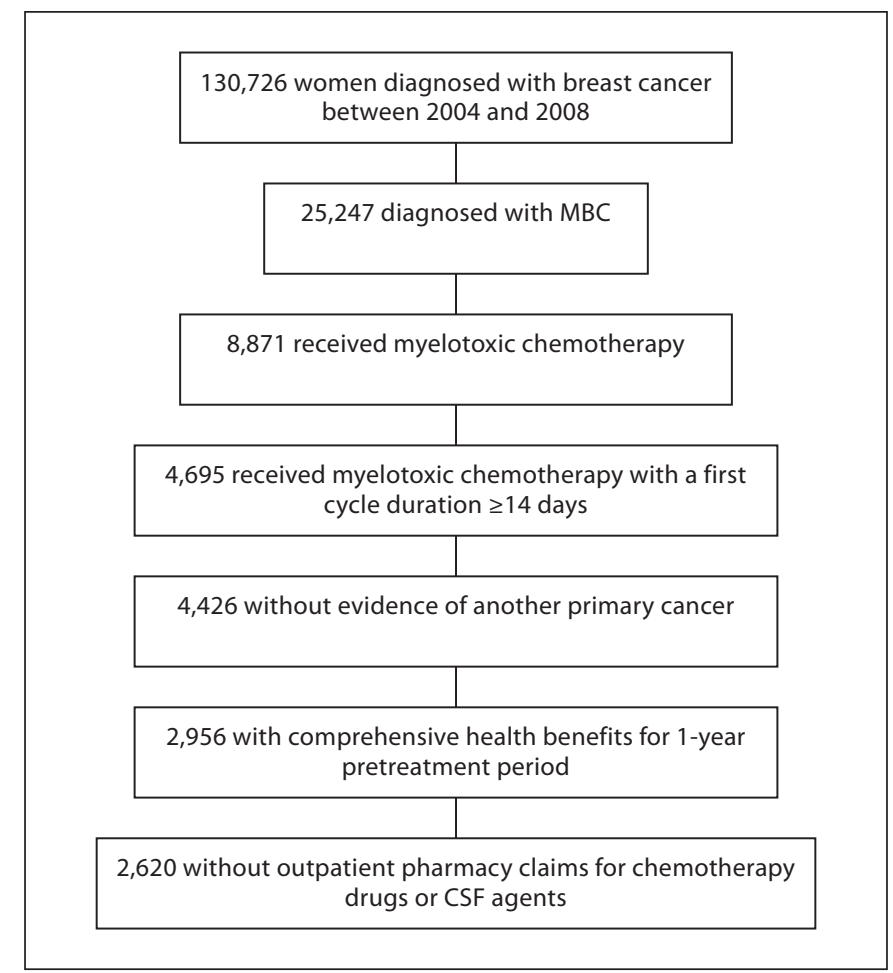

Fig. 1. Selection of study population.

was less than $10 \%$ (table 1). Seventeen percent of subjects had evidence of prior receipt of chemotherapy (i.e. during the 1-year history period), and $10 \%$ had evidence of prior radiation therapy. Other treatment modalities received by study subjects - prior to the index course of chemotherapy - included endocrine therapy (35\%) and trastuzumab/lapatinib (3.5\%). The most common site of metastases was the bone (45\%), followed by the lung (23\%), liver (19\%), and brain/spinal cord (7\%).

\section{Treatment and Supportive Care}

The top five chemotherapy regimens - based on agents administered in cycle 1 - were received by approximately $70 \%$ of all patients, and included doxorubicin/cyclophosphamide (24\%), docetaxel alone (20\%), paclitaxel alone (12\%), carboplatin/paclitaxel (6\%), and carboplatin/docetaxel (5\%) (table 2). From 2004 to 2008, use of doxorubicin/cyclophosphamide decreased from 33 to $27 \%$, while use of docetaxel alone decreased from 35 to $24 \%$; use of paclitaxel alone increased from 10 to $21 \%$ over this same period. Mean $( \pm S D)$ duration of chemotherapy, on an overall basis, was 106 ( \pm 55 ) days, mean number of cycles per course was $5.5( \pm 2.7)$, and mean duration of cycles
Table 1. Patient, cancer and treatment characteristics

\begin{tabular}{|c|c|}
\hline & $\begin{array}{l}\text { Women receiving che- } \\
\text { motherapy for MBC }\end{array}$ \\
\hline Number of patients & 2,620 \\
\hline \multicolumn{2}{|l|}{ Patient } \\
\hline Mean age $\pm S D$, years & $55.1 \pm 10.3$ \\
\hline \multicolumn{2}{|l|}{ Chronic comorbidities, \% } \\
\hline Cardiovascular disease & 4.8 \\
\hline Diabetes & 7.6 \\
\hline Liver disease & 0.3 \\
\hline Renal disease & 0.6 \\
\hline \multicolumn{2}{|l|}{ History of other conditions, $\%$} \\
\hline Anemia & 8.5 \\
\hline Neutropenia & 2.7 \\
\hline Other blood disorders & 4.1 \\
\hline Infection & 25.8 \\
\hline History of hospitalization, \% & 32.1 \\
\hline History of chemotherapy, \% & 16.9 \\
\hline History of radiation therapy, $\%$ & 10.3 \\
\hline \multicolumn{2}{|l|}{ History of other treatments, \% } \\
\hline Endocrine therapy & 34.8 \\
\hline Trastuzumab/lapatinib & 3.5 \\
\hline \multicolumn{2}{|l|}{ Cancer } \\
\hline \multicolumn{2}{|l|}{ Site of metastases* $\%$} \\
\hline Bone & 44.6 \\
\hline Lung & 22.9 \\
\hline Liver & 19.2 \\
\hline Brain and spinal cord & 7.2 \\
\hline Other & 19.7 \\
\hline
\end{tabular}

* Patients may have more than one site of metastasis.

was 19 days $( \pm 8.4)$. Duration of chemotherapy was largely comparable among the most common regimens.

Nearly $50 \%$ of all patients received at least one administration of a CSF agent - for any reason - during their chemotherapy course; $44 \%$ received a CSF as prophylaxis and $17 \%$ received a CSF as treatment (thus, about $11 \%$ $[44+17-50 \%]$ received a CSF as prophylaxis and, at another time during their chemotherapy course, received a CSF as treatment). Pegfilgrastim was the most commonly used CSF agent as primary prophylaxis (91\%) and secondary prophylaxis (75\%), followed by filgrastim (6 and $26 \%$, respectively). Filgrastim was the most commonly used CSF as treatment (68\%), followed by pegfilgrastim (30\%). Among patients who received CSF prophylactically, mean number of cycles with prophylaxis was 3.7 ( \pm 2.2). Prophylaxis was first administered on day 1 of the cycle (i.e., on the same day chemotherapy was first administered in the cycle) for $18 \%$ of patients, day 2 for $62 \%$, 
Table 2. Use of CSF in study population

\begin{tabular}{|c|c|c|c|c|c|c|}
\hline & $\begin{array}{l}\text { Overall } \\
(n=2,620)\end{array}$ & $\begin{array}{l}\text { Doxorubicin/ } \\
\text { cyclophos- } \\
\text { phamide } \\
(\mathrm{n}=641)\end{array}$ & $\begin{array}{l}\text { Docetaxel } \\
(\mathrm{n}=531)\end{array}$ & $\begin{array}{l}\text { Paclitaxel } \\
(\mathrm{n}=316)\end{array}$ & $\begin{array}{l}\text { Carboplatin/ } \\
\text { paclitaxel } \\
(\mathrm{n}=160)\end{array}$ & $\begin{array}{l}\text { Carboplatin/ } \\
\text { docetaxel } \\
(\mathrm{n}=133)\end{array}$ \\
\hline \multicolumn{7}{|l|}{ Chemotherapy course } \\
\hline \multicolumn{7}{|l|}{ Duration, days } \\
\hline Mean $\pm S D$ & $106.0 \pm 55.2$ & $105.5 \pm 46.4$ & $110.0 \pm 61.2$ & $92.5 \pm 59.5$ & $107.7 \pm 60.4$ & $114.1 \pm 51.1$ \\
\hline $\mathrm{Min} / \mathrm{med} / \max$ & $14 / 105 / 364$ & $14 / 103 / 280$ & $14 / 106 / 295$ & $14 / 91 / 274$ & $14 / 105 / 364$ & $14 / 112 / 269$ \\
\hline \multicolumn{7}{|l|}{ Chemotherapy cycles } \\
\hline \multicolumn{7}{|l|}{ Number per course } \\
\hline Mean \pm SD & $5.5 \pm 2.7$ & $6.0 \pm 2.5$ & $5.6 \pm 2.9$ & $5.3 \pm 3.1$ & $5.4 \pm 2.6$ & $5.3 \pm 2.2$ \\
\hline $\mathrm{Min} / \mathrm{med} / \max$ & $1 / 5 / 9$ & $1 / 7 / 9$ & $1 / 6 / 9$ & $1 / 5 / 9$ & $1 / 5 / 9$ & $1 / 5 / 9$ \\
\hline \multicolumn{7}{|l|}{ Duration, days } \\
\hline Mean $\pm S D$ & $18.8 \pm 8.4$ & $19.8 \pm 6.7$ & $19.5 \pm 8.2$ & $15.0 \pm 7.9$ & $19.0 \pm 8.6$ & $21.4 \pm 7.5$ \\
\hline $\mathrm{Min} / \mathrm{med} / \max$ & $7 / 21 / 60$ & $7 / 21 / 60$ & $7 / 21 / 60$ & $7 / 14 / 58$ & $7 / 21 / 58$ & $7 / 21 / 59$ \\
\hline \multicolumn{7}{|l|}{ Duration, in weeks, \% } \\
\hline Weekly ${ }^{1}$ & 17.9 & 1.0 & 16.8 & 34.7 & 22.5 & 8.3 \\
\hline Biweekly & 21.7 & 38.9 & 13.2 & 30.0 & 12.3 & 8.5 \\
\hline Triweekly & 44.7 & 46.9 & 54.6 & 26.5 & 47.1 & 64.2 \\
\hline Other & 15.7 & 13.2 & 15.4 & 8.8 & 18.1 & 19.0 \\
\hline Use of CSF for any reason during course, \% & 49.3 & 60.1 & 36.0 & 30.1 & 52.5 & 65.4 \\
\hline $\begin{array}{l}\text { Use of prophylaxis during course, \% } \\
\text { CSF agent, \% }\end{array}$ & 44.3 & 55.2 & 28.4 & 25.9 & 47.5 & 60.9 \\
\hline Pegfilgrastim & 87.2 & 92.1 & 73.0 & 86.8 & 81.0 & 78.3 \\
\hline Filgrastim & 15.3 & 10.5 & 10.0 & 10.5 & 11.0 & 21.7 \\
\hline Sargramostim & 3.7 & 2.8 & 4.0 & 3.9 & 2.0 & 4.3 \\
\hline \multicolumn{7}{|l|}{ Cycle day of first receipt, $\%$} \\
\hline 1 & 18.0 & 19.2 & 18.3 & 20.2 & 17.5 & 25.9 \\
\hline 2 & 62.1 & 65.9 & 56.7 & 63.6 & 62.7 & 55.3 \\
\hline$\geq 3$ & 19.9 & 14.8 & 25.0 & 16.3 & 19.7 & 18.8 \\
\hline Duration of prophylaxis ${ }^{2}$, mean $\pm S D$ & $4.2 \pm 4.0$ & $6.2 \pm 5.7$ & $3.1 \pm 2.4$ & $3.6 \pm 4.2$ & $4.1 \pm 2.2$ & $5.0 \pm 4.2$ \\
\hline \multicolumn{7}{|l|}{ CSF agent, $\%$} \\
\hline Pegfilgrastim & 91.4 & 92.9 & 85.9 & 94.0 & 97.6 & 91.7 \\
\hline Filgrastim & 6.1 & 5.3 & 12.9 & 2.0 & 2.4 & 3.3 \\
\hline Sargramostim & 3.1 & 2.1 & 1.2 & 6.0 & 0.0 & 5.0 \\
\hline \multicolumn{7}{|l|}{ Cycle day of first receipt, \% } \\
\hline 1 & 16.7 & 18.9 & 20.2 & 14.6 & 19.0 & 17.5 \\
\hline 2 & 65.7 & 68.4 & 65.5 & 68.8 & 69.0 & 54.4 \\
\hline$\geq 3$ & 17.7 & 12.7 & 14.3 & 16.7 & 11.9 & 28.1 \\
\hline Duration of prophylaxis ${ }^{2}$, mean $\pm S D$ & $4.5 \pm 4.1$ & $5.3 \pm 5.1$ & $2.3 \pm 2.6$ & $5.0 \pm 3.3$ & $7.0 \pm 0.0$ & $8.2 \pm 5.8$ \\
\hline $\begin{array}{l}\text { Use of secondary prophylaxis, \% } \\
\text { CSF agent, \% }\end{array}$ & 13.2 & 11.4 & 12.4 & 10.1 & 21.2 & 15.8 \\
\hline Pegfilgrastim & 75.4 & 82.2 & 75.8 & 81.3 & 73.5 & 81.0 \\
\hline Filgrastim & 26.4 & 19.2 & 22.7 & 28.1 & 17.6 & 23.8 \\
\hline Sargramostim & 4.1 & 5.5 & 3.0 & 0.0 & 8.8 & 0.0 \\
\hline $\begin{array}{l}\text { No. of cycles with prophylaxis, mean } \pm \text { SD } \\
\text { Cycle day of first receipt, } \%\end{array}$ & $3.4 \pm 1.9$ & $3.7 \pm 1.9$ & $3.4 \pm 2.2$ & $3.3 \pm 2.3$ & $3.1 \pm 1.8$ & $3.7 \pm 1.7$ \\
\hline 1 & 18.3 & 19.4 & 18.0 & 20.8 & 17.2 & 27.6 \\
\hline 2 & 61.3 & 65.0 & 55.4 & 63.0 & 61.3 & 55.5 \\
\hline$\geq 3$ & 20.4 & 15.6 & 26.6 & 16.2 & 21.5 & 17.0 \\
\hline Duration of prophylaxis ${ }^{2}$, mean $\pm S D$ & $4.1 \pm 4.0$ & $6.6 \pm 6.0$ & $3.2 \pm 2.4$ & $3.5 \pm 4.3$ & $4.0 \pm 2.1$ & $4.3 \pm 3.6$ \\
\hline
\end{tabular}


Table 2 (continued)

\begin{tabular}{|c|c|c|c|c|c|c|}
\hline & $\begin{array}{l}\text { Overall } \\
(\mathrm{n}=2,620)\end{array}$ & $\begin{array}{l}\text { Doxorubicin/ } \\
\text { cyclophos- } \\
\text { phamide } \\
(\mathrm{n}=641)\end{array}$ & $\begin{array}{l}\text { Docetaxel } \\
(\mathrm{n}=531)\end{array}$ & $\begin{array}{l}\text { Paclitaxel } \\
(\mathrm{n}=316)\end{array}$ & $\begin{array}{l}\text { Carboplatin/ } \\
\text { paclitaxel } \\
(\mathrm{n}=160)\end{array}$ & $\begin{array}{l}\text { Carboplatin/ } \\
\text { docetaxel } \\
(n=133)\end{array}$ \\
\hline $\begin{array}{l}\text { Treatment, \% } \\
\quad \text { CSF agent, \% }\end{array}$ & 16.8 & 16.5 & 16.9 & 8.2 & 20.0 & 17.3 \\
\hline Pegfilgrastim & 30.2 & 32.1 & 26.7 & 34.6 & 31.3 & 43.5 \\
\hline Filgrastim & 68.0 & 67.0 & 74.4 & 69.2 & 56.3 & 52.2 \\
\hline Sargramostim & 9.1 & 10.4 & 2.2 & 3.8 & 15.6 & 8.7 \\
\hline $\begin{array}{l}\text { No. of cycles with treatment, mean } \pm S D \\
\text { Cycle day of first receipt, } \%\end{array}$ & $1.8 \pm 1.4$ & $1.8 \pm 1.4$ & $2.1 \pm 1.7$ & $1.7 \pm 1.0$ & $1.7 \pm 1.3$ & $1.6 \pm 1.0$ \\
\hline 6 & 19.7 & 20.5 & 15.3 & 15.6 & 20.8 & 25.6 \\
\hline 7 & 11.5 & 5.1 & 17.7 & 10.9 & 4.2 & 5.1 \\
\hline$\geq 8$ & 68.8 & 74.4 & 67.0 & 73.4 & 75.0 & 69.2 \\
\hline Duration of treatment ${ }^{2}$, mean $\pm S D$ & $2.8 \pm 2.7$ & $3.2 \pm 3.8$ & $2.7 \pm 2.2$ & $2.6 \pm 2.7$ & $2.4 \pm 1.7$ & $1.9 \pm 1.3$ \\
\hline
\end{tabular}

${ }^{1}$ Patients may have received cycles $<14$ days in duration after the first cycle.

${ }^{2}$ Duration of receipt based only on data for filgrastim and sargramostim (i.e. the daily agents).

and day 3 or later for $20 \%$. Mean duration of prophylaxis with the daily agents (i.e. filgrastim and sargramostim) was $4.2( \pm 4.0)$ days.

Thirty-one percent of all subjects (70\% of subjects receiving prophylaxis at anytime) received CSF prophylaxis in their first chemotherapy cycle, and an additional $13 \%$ of all subjects ( $30 \%$ of subjects receiving prophylaxis at any time) first received CSF prophylaxis after cycle one. Receipt of primary prophylaxis was highest among patients receiving chemotherapy with carboplatin/docetaxel (45\%) and lowest among those receiving chemotherapy with docetaxel alone or paclitaxel alone (16\%); receipt of secondary prophylaxis was largely comparable across regimens.

\section{Risk and Burden of Neutropenic Complications}

Over their course of chemotherapy, 286 (11.0\% [95\% CI 9.8-12.2] of all subjects) patients experienced CINC, the large majority $(88 \%)$ of whom required inpatient care (9.7\% [8.6-10.9] of all subjects) (table 3). Risk of CINC was $4.9 \%$ [4.1-5.8] in cycle 1 (45\% of all subjects experiencing complications), and was $2.3 \%(1.8-2.9)$ in cycle 2 (21\% of all subjects experiencing complications). In all subsequent cycles, 4.9\% (4.1-5.8) of patients experienced CINC requiring inpatient care or outpatient care only. Overall, $16.6 \%$ of patients were hospitalized for any reason during their chemotherapy course.

Among the patients who developed CINC requiring inpatient care, mean (95\% CI) associated expenditures were USD 12,869 (USD 12,622-13,116), comprising USD 12,554 (USD 12,307-12,801) for hospital-related care and USD 315 (USD 247-383) for subsequent outpatient care during the same cycle (table 4). Mean number of days in hospital was $6.6(1.8-11.3)$. Among the patients who developed CINC requiring outpatient care only, mean associated expenditures were USD 2,030 (USD 1,9252,135 ), comprising USD 1,249 (USD 1,146-1,351) for outpatient visits for reasons other than the receipt of CSF or antimicrobial therapy, USD 443 (USD 372-513) for receipt of CSF and USD 338 (USD 295-381) for receipt of antimicrobial therapy. The mean number of outpatient encounters for CINC was 2.9 (95\% CI 0.0-5.8).

\section{Discussion}

Using data from 2 large U.S. private healthcare claims databases, we examined the risk and burden of CINC among women with MBC. Not surprisingly, our study population comprised women who had additional risk factors for complications, including a history of chemotherapy or radiation therapy, chronic comorbidities and/ or bone marrow involvement. We found that over $10 \%$ of these women experienced CINC during their chemotherapy course, most commonly requiring inpatient care and during the first cycle. We also found that the economic consequences were substantial for the patients experiencing neutropenic events, with mean associated costs ap- 
Table 3. Risk of neutropenic complications

\begin{tabular}{|c|c|c|c|c|c|c|}
\hline & $\begin{array}{l}\text { Overall } \\
(\mathrm{n}=2,620)\end{array}$ & $\begin{array}{l}\text { Doxorubicin/ } \\
\text { cyclophosphamide } \\
(\mathrm{n}=641)\end{array}$ & $\begin{array}{l}\text { Docetaxel } \\
(\mathrm{n}=531)\end{array}$ & $\begin{array}{l}\text { Paclitaxel } \\
(\mathrm{n}=316)\end{array}$ & $\begin{array}{l}\text { Carboplatin/ } \\
\text { paclitaxel } \\
(\mathrm{n}=160)\end{array}$ & $\begin{array}{l}\text { Carboplatin/ } \\
\text { docetaxel } \\
(\mathrm{n}=133)\end{array}$ \\
\hline \multicolumn{7}{|c|}{ Risk of neutropenic complications during course, $\%(95 \%$ CI) } \\
\hline All events & $11.0(9.8-12.2)$ & $9.7(7.5-12.2)$ & $11.5(8.9-14.5)$ & $7.6(4.9-11.1)$ & $5.6(2.6-10.4)$ & $9.8(5.3-16.1)$ \\
\hline Requiring inpatient care & $9.7(8.6-10.9)$ & $8.6(6.5-11.0)$ & $9.8(7.4-12.6)$ & $7.3(4.7-10.7)$ & $5.0(2.2-9.6)$ & $7.5(3.7-13.4)$ \\
\hline Requiring outpatient care only & $1.6(1.2-2.2)$ & $1.2(0.5-2.4)$ & $1.7(0.8-3.2)$ & $0.6(0.1-2.3)$ & $0.6(0.0-3.4)$ & $2.3(0.5-6.5)$ \\
\hline \multicolumn{7}{|c|}{ Risk of neutropenic complications during cycle 1, \% (95\% CI) } \\
\hline All events & $4.9(4.1-5.8)$ & $3.7(2.4-5.5)$ & $4.1(2.6-6.2)$ & $2.8(1.3-5.3)$ & $2.5(0.7-6.3)$ & $3.8(1.2-8.6)$ \\
\hline Requiring inpatient care & $4.5(3.7-5.3)$ & $3.4(2.2-5.2)$ & $3.6(2.2-5.5)$ & $2.8(1.3-5.3)$ & $2.5(0.7-6.3)$ & $3.8(1.2-8.6)$ \\
\hline Requiring outpatient care only & $0.5(0.2-0.8)$ & $0.3(0.0-1.1)$ & $0.6(0.1-1.6)$ & $0.0(0.0-1.2)$ & $0.0(0.0-2.3)$ & $0.0(0.0-2.7)$ \\
\hline \multicolumn{7}{|c|}{ Risk of neutropenic complications during cycle 2, \% (95\% CI) } \\
\hline All events & $2.3(1.8-2.9)$ & $1.6(0.8-2.9)$ & $2.8(1.6-4.6)$ & $1.3(0.3-3.2)$ & $1.9(0.4-5.4)$ & $2.3(0.5-6.5)$ \\
\hline Requiring inpatient care & $1.8(1.3-2.4)$ & $1.2(0.5-2.4)$ & $2.3(1.2-3.9)$ & $1.3(0.3-3.2)$ & $1.3(0.2-4.4)$ & $1.5(0.2-5.3)$ \\
\hline Requiring outpatient care only & $0.5(0.3-0.8)$ & $0.3(0.0-1.1)$ & $0.6(0.1-1.6)$ & $0.0(0.0-1.2)$ & $0.6(0.0-3.4)$ & $0.8(0.0-4.1)$ \\
\hline \multicolumn{7}{|c|}{ Risk of neutropenic complications during cycles 3-9, \% (95\% CI) } \\
\hline All events & $4.9(4.1-5.8)$ & $5.1(3.6-7.2)$ & $4.9(3.2-7.1)$ & $3.8(2.0-6.5)$ & $1.9(0.4-5.4)$ & $5.3(2.1-10.5)$ \\
\hline Requiring inpatient care & $4.2(3.5-5.0)$ & $4.5(3.1-6.4)$ & $4.3(2.8-6.4)$ & $3.2(1.5-5.7)$ & $1.9(0.4-5.4)$ & $3.8(1.2-8.6)$ \\
\hline Requiring outpatient care only & $0.8(0.5-1.2)$ & $0.6(0.2-1.6)$ & $0.6(0.1-1.6)$ & $0.6(0.1-2.3)$ & $0.0(0.0-2.3)$ & $1.5(0.2-5.3)$ \\
\hline
\end{tabular}

For patients with multiple events, only the first one during the corresponding period was considered in these analyses.

proaching USD 13,000 for those requiring inpatient care and totaling over USD 2,000 for those requiring outpatient care only.

Our findings on the risk of CINC among women with $\mathrm{MBC}$ are largely comparable to published data from clinical trials, despite the differences in study designs and case-ascertainment criteria. For example, among women in our study population who received chemotherapy with doxorubicin/cyclophosphamide - the most common regimen - the number requiring inpatient care for CINC (9.7\%) was similar to that ( 9 and $10 \%$, respectively) in two randomized controlled trials of the same combination of chemotherapy agents as first-line treatment in MBC [27, 28]. Our findings also are largely comparable to those from a recent retrospective cohort study focusing on patients with advanced non-small cell lung cancer. In the Stokes et al. study [21], 10\% of the subjects were classified as having developed febrile neutropenia. Of these, $72 \%$ required hospitalization; corresponding estimates from our study were 11 and $88 \%$. The percentage of MBC patients developing CINC who required inpatient care in our study also is consistent with data from another published study of CSF use in cancer patients receiving chemotherapy [29, 30]. In contrast with rates for lung cancer and breast cancer, a population-based examination of the hospitalization rate for chemotherapy-associated neutropenia among older adults with non-Hodgkin's lymphoma in the US reported that $22 \%$ of subjects were hospitalized [31].
Table 4. Burden of neutropenic complications

\begin{tabular}{lc}
\hline & Value $(95 \% \mathrm{CI})$ \\
\hline $\begin{array}{l}\text { All neutropenic complications }(\mathrm{n}=342) \\
\text { Expenditures, USD }\end{array}$ & $11,094(10,853-11,335)$ \\
\hline $\begin{array}{l}\text { Events requiring inpatient care }(\mathrm{n}=286) \\
\text { Hospital days }\end{array}$ & $6.6(1.8-11.3)$ \\
Expenditures, USD & $12,554(12,307-12,801)$ \\
$\quad$ Inpatient & $315(247-383)$ \\
Outpatient & $12,869(12,622-13,116)$ \\
Total & $56)$ \\
\hline Events requiring outpatient care only $(\mathrm{n}=$ & $2.9(0.0-5.8)$ \\
Number of encounters & $1,249(1,146-1,351)$ \\
Expenditures, USD & $443(372-513)$ \\
Encounters & $338(295-381)$ \\
CSF & \\
Antibiotics & $2,030(1,925-2,135)$ \\
Total &
\end{tabular}

For patients with multiple events during the chemotherapy course, each one was considered separately in these analyses.

Interestingly, while nearly $50 \%$ of patients received CSF agents as prophylaxis or treatment during their chemotherapy course - findings comparable to those reported by Stokes et al. [21, 26] - it appears that many of the patients who received prophylaxis in our study were ad- 
ministered these agents in a manner that is inconsistent with recommendations. Nearly one fifth of all patients receiving prophylaxis (primary or secondary) first received CSF on day one of the cycle, while recommendations specify that prophylaxis should be initiated $24-72 \mathrm{~h}$ after completion of chemotherapy and that 'administration of growth factor on same day as chemotherapy is not recommended' [26]. Moreover, among patients receiving one of the CSF agents requiring daily administration (filgrastim or sargramostim), the mean duration of prophylaxis was only about 4 days, substantially lower than the 10-11 days typically required for post-nadir absolute neutrophil count (ANC) recovery in two large recent clinical trials $[32,33]$. We note, however, that such patients represent only a minority of patients who received prophylaxis as the large majority received prophylaxis with pegfilgrastim, which requires only a single dose per cycle.

Our estimates of the cost of CINC were similar to those previously reported for other patient populations $[10,21,22,34]$. In two of these studies, which utilized hospital discharge records, the mean cost of hospitalization among patients with solid tumors ranged from USD 8,100 to USD 13,354 , and for the subgroup of patients with breast cancer (all stages) mean cost ranged from USD 7,100 to USD 12,372 [10, 22]. In the aforementioned study focusing on patients with advanced non-small cell lung cancer, mean total cost of neutropenia-related care for those treated on an inpatient or outpatient basis was estimated to be USD 12,148, including USD 7,598 for inpatient care [21]. We note that our estimates of the economic burden of CINC may not reflect the full impact of this condition, since downstream-associated (i.e. post-index cycle) costs were not captured, and such costs have been found to represent a substantial proportion of the total economic burden of this condition [35].

Potential biases with regard to patient (treatment) selection and outcome measurement may impact this study. Most important, the accuracy of the algorithms for identifying patients receiving treatment for $\mathrm{MBC}$ and for identifying CINC is unknown, as they have not been validated. Because an ICD-9-CM diagnosis code for CINC (i.e. neutropenia-related fever or infection) does not exist, an algorithm based on codes for neutropenia, fever and infection (and i.v. antibiotic therapy for outpatient care) was employed to identify encounters for the events of interest. While this algorithm has not been formally validated, it (and similar ones) has been utilized in a number of studies published to date [35-37]. Although our algorithm for ascertaining outpatient treatment of CINC required the administration of i.v. antibiotic therapy, we ac- knowledge that some low-risk FN patients may receive outpatient management with oral (rather than i.v.) antimicrobial therapy in clinical practice. We suspect, however, that the large majority of patients who receive oral antimicrobial drugs on an outpatient basis have either a low ANC (i.e. neutropenia) and are receiving the drugs as prophylaxis against $\mathrm{FN}$, or a normal ANC and are receiving the drugs for a coded infection. To the extent that we may have missed such patients, we may have underestimated the frequency of outpatient FN and may have - to the extent that these patients differ systematically in their economic profile from outpatient FN cases treated with IV agents - mis-estimated economic cost. Finally, while some infection-related encounters during a given cycle may not be related to the neutropenic event (especially those that occur temporally distal, e.g. 2 weeks), it is likely that most of these encounters are a consequence of treatment and the associated neutropenia.

Several generic limitations of retrospective studies based on healthcare claims data also should be noted. All such databases contain errors of omission and commission in coding. In addition, certain biases in coding may exist, such that patients who, for example, are hospitalized for CINC may be more likely to have 'neutropenia' (ICD-9-CM 288.0) designated as a secondary (or even primary) diagnosis on future encounters as opposed to patients without a history of these complications, all else being equal. Moreover, information often is not available for one or more clinically important parameters (e.g. in our study, ANCs and performance status), and pertinent medical history can be left-censored (e.g. receipt of chemotherapy and neutropenic complications occurring before the time period of the study database are unobservable). Thus, as noted above, although most index courses of chemotherapy probably represent the first course of treatment subsequent to the diagnosis of MBC, we cannot be certain that this was the case for all patients in the study population. The impact of these limitations on our results cannot be assessed within the scope of this study.

In addition to these generic limitations of healthcare claims data, several limitations specific to our study should be noted. First, for some patients, an HCPCS Level I code for the administration of chemotherapy would have been present without any HCPCS Level II codes for specific chemotherapy agents. Not knowing whether or not the agent-specific codes had been omitted erroneously, the HCPCS Level I code had been incorrectly entered or chemotherapy had been obtained from another source (e.g. investigational drug study), we excluded such pa- 
tients (i.e. those with missing drug information) from analyses. Second, chemotherapy regimens may be misclassified for patients who received combination therapy with a taxane or anthracycline agent and a new chemotherapy agent during the period in which a unique code is not available. However, only a handful of new myelosuppressive chemotherapy agents were approved for use in the USA during the period of interest, and all such agents were either infrequently used in clinical practice or not indicated for use as combination therapy with a taxane or anthracycline agent in the treatment of MBC. Third, patients obtaining chemotherapy or CSF agents via the retail pharmacy were excluded from analyses since the precise dates of administration cannot be determined in healthcare claims databases. Fourth, our study sample was comprised principally of cancer patients with private healthcare coverage who were less than 65 years old. Our study population may thus not have been representative of the entire population of women with MBC in the USA, which would limit the generalized application of its findings.

In summary, the results of this study suggest that the incidence of CINC among women with MBC is high and that the economic consequences of this condition are considerable. Additional research is needed to identify the subgroups of women with $\mathrm{MBC}$ who are at a particularly high risk of CINC, so that they may be targeted for preventative measures.

\section{Acknowledgments}

Funding for this research was provided by Amgen Inc. to Policy Analysis Inc. We thank Martha Mutomba from Amgen Inc. for writing assistance.

\section{References}

1 American Cancer Society, Inc., Surveillance and Health Policy Research. http:www.cancer.org (accessed November 29, 2009).

2 Honig SF: Hormonal therapy and chemotherapy; in Harris JR, Morrow M, Lippman ME, et al (eds): Diseases of the Breast. Lippincott-Raven, Philadelphia, 1996, pp 669734

-3 Glass AG, Lacey JV, Carreon JD, Hoover RN: Breast cancer incidence, 1980-2006: combined roles of menopausal hormone therapy, screening mammography, and estrogen receptor status. J Natl Cancer Inst 2007;99: $1152-1162$.

4 Rastelli F, Crispino S: Factors predictive of response to hormone therapy in breast cancer. Tumori 2008;94:370-383.

5 Hortobagyi GN: Treatment of breast cancer. N Engl J Med 1998;339:974-984.

6 Higgins MJ, Wolff AC: Therapeutic options in the management of metastatic breast cancer. Oncology (Williston Park) 2008;22:614629.

7 Isakoff S: Triple-negative breast cancer: role of specific chemotherapy agents. Cancer J 2010;16:53-61.

8 Wilcken N, Hornbuckle J, Ghersi D: Chemotherapy alone versus endocrine therapy alone for metastatic breast cancer. Cochrane Database Syst Rev 2003;2:CD002747.

-9 Conlin AK, Seidman AD: Beyond cytotoxic chemotherapy for the first-line treatment of HER2-negative, hormone-insensitive metastatic breast cancer: current status and future opportunities. Clin Breast Cancer 2008;8: 215-223.

10 Caggiano V, Weiss RV, Rickert TS, LindeZwirble WT: Incidence, cost and mortality of neutropenia hospitalization associated with chemotherapy. Cancer 2005;103:1916-1924.

11 Smith TJ, Khatcheressian J, Lyman GH, Ozer H, Armitage JO, Balducci L, et al: 2006 update of recommendations for the use of white blood cell growth factors: an evidence-based clinical practice guideline. J Clin Oncol 2006;24:3187-205.

12 Bonadonna G, Moliterni A, Zambetti M Daidone MG, Pilotti S, Gianni L, et al: 30 years' follow up of randomised studies of adjuvant CMF in operable breast cancer: cohort study. BMJ 2005;330:217.

13 Lyman GH, Dale DC, Crawford J: Incidence and predictors of low-dose intensity in adjuvant breast cancer chemotherapy: a nationwide study of community practices. J Clin Oncol 2003;21:4524-4531.

14 Kwak LW, Halpern J, Olshen RA, Horning SJ: Prognostic significance of actual dose intensity in diffuse large-cell lymphoma: results of a tree-structured survival analysis. J Clin Oncol 1990;8:963-977.

15 Cardoso F, Bedard PL, Winer EP, et al: International guidelines for management of metastatic breast cancer: combination versus sequential single-agent chemotherapy. J Natl Cancer Inst 2009;101:1174-1181.

16 Mao W, Guan X, Tucker S, et al: Second-line combination chemotherapy with vinorelbine and capecitabine in patients with advanced breast cancer previously treated with anthracyclines and/or taxanes. Chemotherapy 2011;57:71-76.

17 Taguchi T, Nakayama T, Masuda N, et al: Study of low-dose capecitabine monotherapy for metastatic breast cancer. Chemotherapy $2010 ; 56: 166-170$.
18 Somali I, Alacacioglu A, Tarhan M, et al: Cisplatin plus gemcitabine chemotherapy in taxane/anthracycline-resistant metastatic breast cancer. Chemotherapy 2009;55:155-160.

19 Karnon J, Kerr GR, Jack W, Papo NL, Cameron DA: Health care costs for the treatment of breast cancer recurrent events: estimates from a UK-based patient-level analysis. Br J Cancer 2007;97:479-485.

20 Smith TJ, Hillner BE: Bending the cost curve in cancer care. N Engl J Med 2011;364:20602065

-21 Stokes ME, Muehlenbein CE, Marciniak $\mathrm{MD}$, et al: Neutropenia-related costs in patients with first-line chemotherapy for advanced non-small cell lung cancer. J Manag Care Pharm 2009;15:669-682.

22 Kuderer NM, Dale DC, Crawford J, Cosler LE, et al: Mortality, morbidity, and cost associated with febrile neutropenia in adult cancer patients. Cancer 2006; 106:22582266

23 Lyman GH, Kuderer N, Greene J, Balducci L: The economics of febrile neutropenia: implications for the use of colony-stimulating factors. Eur J Cancer 1998;34:1857-1864

24 Code of Federal Regulations: Title 45 Public Welfare, Part 46: Protection of Human Subjects. 46.101.

25 Stokes ME, Thompson D, Montoya EL, et al: Ten-year survival and cost following breast cancer recurrence: estimates from SEERMedicare data. Value Health 2008;11:213-220.

26 National Comprehensive Cancer Network: NCCN clinical practice guidelines in oncology: myeloid growth factors, v1.2010. http:// www.nccn.org/professionals/physician_gls/ $\mathrm{PDF} /$ myeloid_growth.pdf. 
-27 Biganzoli L, Cufer T, Coleman BR, et al: Doxorubicin and paclitaxel versus doxorubicin and cyclophosphamide as first-line chemotherapy in metastatic breast cancer: the European Organization for Research and Treatment of Cancer 10961 multicenter phase III trial. J Clin Oncol 2002;20:31143121.

-28 Nabholtz JM, Falkson C, Campos D, et al: Docetaxel and doxorubicin compared with doxorubicin and cyclophosphamide as firstline chemotherapy for metastatic breast cancer: results of a randomized, multicenter, phase III trial. J Clin Oncol 2003;21:968-975.

-29 Cosler LE, Sivasubramaniam V, Agboola O, et al: Effect of outpatient treatment of febrile neutropenia on the risk threshold for the use of CSF in patients with cancer treated with chemotherapy. Value Health 2005;8:47-52.

-30 Ramsey SD, Liu Z, Boer R, et al: Cost-effectiveness of primary versus secondary prophylaxis with pegfilgrastim in women with early-stage breast cancer receiving chemotherapy. Value Health 2009;12:217-225.
31 Chen-Hardee S, Chrischilles EA, Voelker $\mathrm{MD}$, et al: Population-based assessment for hospitalizations for neutropenia from chemotherapy in older adults with non-Hodgkin's lymphoma (United States). Cancer Causes Control 2006;17:647-654.

32 Green MD, Koelbl H, Baselga J, et al: A randomized double-blind multicenter phase III study of fixed-dose single-administration pegfilgrastim versus daily filgrastim in patients receiving myelosuppressive chemotherapy. Ann Oncol 2003;14:29-35.

33 Holmes FA, Jones SE, O’Shaughnessy J, et al: Comparable efficacy and safety profiles of once-per-cycle pegfilgrastim and daily injection filgrastim in chemotherapy-induced neutropenia: a multicenter dose-finding study in women with breast cancer. Ann Oncol 2002;13:903-909.
34 Elting LS, Lu C, Escalante CP, et al: Outcomes and cost of outpatient or inpatient management of 712 patients with febrile neutropenia. J Clin Oncol 2008;26:606-611.

35 Weycker D, Malin J, Edelsberg J, Glass A, Gokhale M, Oster G: Cost of neutropenic complications of chemotherapy. Ann Oncol 2008;19:454-460.

>36 Weycker D, Malin J, Kim J, Barron R, Edelsberg J, Kartashov A, et al: Risk of hospitalization for neutropenic complications of chemotherapy in patients with primary solid tumors receiving pegfilgrastim or filgrastim prophylaxis: a retrospective cohort study. Clin Ther 2009;31:1069-1081.

>37 Heaney ML, Toy EL, Vekeman F, Laliberte F, Dority BL, Perlman D, et al: Comparison of hospitalization risk and associated costs among patients receiving sargramostim, filgrastim, and pegfilgrastim for chemotherapy-induced neutropenia. Cancer 2009;115: 4839-4848. 